\title{
Liberalisierung in der Elektrizitätswirtschaft: Zuspitzung der Verteilungskonflikte
}

\author{
Heinz-J. Bontrup, Ralf-M. Marquardt \\ Werner Voß
}

\begin{abstract}
Wie sich die Liberalisierung auf Konzentrationsprozesse, Preise und Gewinne der Elektrizitätsversorgungsunternehmen (EVUs) ausgewirkt hat, ist in zahlreichen Studien bereits untersucht und öffentlich diskutiert worden. Aufregung lösten insbesondere die Strompreiserhöhungen der letzten beiden Jahre aus. Die Umweltfrage dagegen rückte angesichts der erschreckenden Klimaszenarien erst jüngst wieder in den Blickpunkt. Gänzlich unterbelichtet aber blieb die hier behandelte Frage, welche Folgen die bisherige Öffnung für die Beschäftigten und den Verteilungskonflikt innerhalb der EVUs hatte und welche Konsequenzen durch die „Anreizregulierung“ und die Novelle des Gesetzes gegen Wettbewerbsbeschränkungen (GWB) noch zu erwarten sind. ${ }^{1}$
\end{abstract}

\section{Bisherige Wirkungen des Liberalisierungsprozesses}

In der Stromversorgung in Deutschland gab es aufgrund von unterstellten Subadditivitäten ${ }^{2}$ bei der Erzeugung und Verteilung von Strom lange Zeit natürliche Monopole (Schmidt 2005, S. 36ff.). Eine derart prominente Marktstellung hatten sowohl im Kraftwerks- als auch im Netzbereich - abgesichert durch Demarkationsverträge die sogenannten Gebietsmonopolisten in Form von neun vertikal marktintegrierten Verbundunternehmen inne: RWE, PreuBenElektra, Bayernwerk, VEAG, Badenwerke AG, EVS, VEW, HEW und BEWAG. Sie befanden sich teils im privaten, teils im staatlichen Eigentum. Hinzu kamen Regionalversorger sowie auf der dritten Marktstufe dezentral aufgestellte kommunale Stadtwerke. Alle Energieversorger unterlagen im Tarifkundenbereich einer staatlichen Preisaufsicht und wurden im Sonderkundenbereich durch das Bundeskartellamt bzw. die jeweiligen Landeskartellbehörden einer Preiskontrolle unterzogen (Bontrup/Troost 1988).

Getragen von der Idealvorstellung eines liberalen Marktwettbewerbs versucht die EU-Kommission nun seit etwas mehr als einem Jahrzehnt, die Strukturen aufzubrechen und mit Unterstützung der nationalen Regierungen einen „Binnenmarkt für Energie“ in Europa zu schaffen. Aus dem Grundsatz der Warenverkehrsfreiheit leitete sie mit der Richtlinie Elektrizität 96/92/ EG die Öffnung der Strommärkte in Europa ein. Bis Mitte 2007 sollten die Märkte für Privatkunden geöffnet sein, also jeder Ver- braucher seinen Stromversorger frei wählen können. Dieses Ziel ist mittlerweile in allen EU 15-Staaten realisiert.

Auch in Deutschland erfolgte 1998 eine Novellierung des noch aus dem Jahr 1935 stammenden Energiewirtschaftsgesetzes (EnWG). Die Gebietsmonopole wurden aufgelöst, um Wettbewerb zu schaffen. Ziel war insbesondere, die Strompreise zu senken. Die deutsche Politik verzichtete allerdings auf eine eigentumsrechtliche Entflechtung der schon damals hoch konzentrierten Markt- und Unternehmensstrukturen. Die Initiierung des Wettbewerbs ging so von Anfang an mit ungleichen Startbedingungen einher: „Die schlagartige Öffnung des Wettbewerbs zwischen den Goliaths der Verbundmonopole und der Vielzahl von Davids verstärkte die Vorteile für die Großen" (Hennicke/Müller 2005, S. 132). So standen sich im Jahre 1998 auf einem entfesselten Markt, der in der Vergangenheit Überkapazitäten aufgebaut hatte, schlagartig Konkurrenten mit völlig unterschiedlichen Größenordnungen in den Kraftwerksund Netzkapazitäten sowie einer unterschiedlich breiten Produktpalette (Strom, Gas, Wasser) gegenüber. Die vorhandenen enormen Machtdisparitäten unter den EVUs wurden alsbald durch Konzentrationsprozesse nochmals befördert und bremsten den erhofften Wettbewerb aus.

Ermöglicht wurde eine derartige Entwicklung auch, weil die Politik mit Blick auf den Netzzugang zunächst auf eine mehrmals angepasste „Verbändevereinbarung"gesetzt hatte, die zwischen den Stromanbietern und -nachfragern sowohl die Höhe der Abrechnung der Netzentgelte als auch die technischen Rahmenbedingungen zur Sicherung der Versorgung mit Strom regeln sollte. Integrierte Netzbetreiber, die zugleich Stromproduzenten sind, zeigten jedoch wenig Interesse, über faire Durchleitungsgebühren Mitkonkurrenten der

\footnotetext{
1 Der Artikel basiert auf Erkenntnissen, die im Rahmen des von der Hans-Böckler-Stiftung geförderten Projektes über „Neue Unternehmensstrategien und Mitbestimmungskulturen angesichts liberalisierter Rahmenbedingungen in der Elektrizitätswirtschaft" gewonnen wurden. Das Projekt stützt sich vorrangig auf die Auswertung branchenspezifischer Daten sowie auf eine repräsentative Befragung von Mitbestimmungsträgern und auf Einzelinterviews in den Unternehmen der Elektrizitätswirtschaft. Das über zwei Jahre laufende Projekt wird im Frühjahr 2009 abgeschlossen werden.

2 Subadditivitäten liegen vor, wenn ein (Groß-)Unternehmen die Nachfrage nach einem Gut kostengünstiger bedienen kann als mehrere kleine Unternehmen.
}

Heinz-J. Bontrup, Prof. Dr., Hochschullehrer
für Allgemeine Betriebswirtschaftslehre,
insbesondere für Arbeitsökonomik, an der
FH Gelsenkirchen. Arbeitsschwerpunkte:
Allgemeine Wirtschaftspolitik, Personalwirt-
schaft, Mitbestimmung, Energiepolitik.
e-mail: heinz-josef.bontrup@fh-
gelsenkirchen.de
Ralf-M. Marquardt, Prof. Dr., Hochschul-
lehrer für Volkswirtschaftslehre und Quanti-
tative Methoden an der FH Gelsenkirchen.
Arbeitsschwerpunkte: Allgemeine Wirt-
schaftspolitik, Währungspolitik, Finanz-
märkte, Energiepolitik.
e-mail: ralf-michael.marquardt@fh-
gelsenkirchen.de
Werner Voß, Dipl. Ökonom, Wissenschaft-
licher Projektmitarbeiter. Arbeitsschwer-
punkte: Energiewirtschaft, Chemische
Industrie/Chemieparks, Mitbestimmung
und neue Entgeltsysteme.
e-mail: werner.voss@fh-gelsenkirchen.de




\begin{tabular}{|c|c|c|c|c|}
\hline & E.ON AG & RWE AG & Vattenfall Europe AG & $\begin{array}{c}\text { EnBW Energie } \\
\text { Baden-Württemberg AG }\end{array}$ \\
\hline Jahr des Zusammenschlussses & 2000 & 2000 & 2002 & 1997 \\
\hline Vorgängergesellschaften & $\begin{array}{l}\text { - Vereinigte Elektrizitäts- } \\
\text { und BergwerksAG } \\
\text { (VEBA; Strom: Preussen } \\
\text { Elektra AG, Hannover) } \\
\text { - Vereinigte Industrie- } \\
\text { unternehmungen (VIAG, } \\
\text { davon Strom: Bayernwerk } \\
\text { AG, München) }\end{array}$ & $\begin{array}{l}\text { - RWE Energie AG, Essen } \\
\text { - Vereinigte Elektrizitäts- } \\
\text { werke Westfalen AG, } \\
\text { Dortmund }\end{array}$ & $\begin{array}{l}\text { - VEAG Vereinigte } \\
\text { Energiewerke AG, Berlin } \\
\text { - Hamburgische Electrizitäts- } \\
\text { Werke AG, Hamburg } \\
\text { - inkl. LAUBAG Lausitzer } \\
\text { Braunkohle AG } \\
\text { - Berliner Kraft- und } \\
\text { Licht(Bewag)-AG, Berlin }\end{array}$ & $\begin{array}{l}\text { - Badenwerk AG, Karlsruhe } \\
\text { - Energie-Versorgung } \\
\text { Schwaben AG (EVS), } \\
\text { Stuttgart } \\
\text { - } 2003 \text { kommt die Neckar- } \\
\text { werke Stuttgart AG hinzu }\end{array}$ \\
\hline Kunden in Millionen (2005) & Strom: 22, Gas: 8 & Strom: 20, Gas: 10 & Strom: 2,9 & Strom: 5 Gas: 0,4 \\
\hline Stromabsatz 2006 in Mrd. kWh & rund 400 & 312 & 153 & 119 \\
\hline Umsatz in Mio. Euro & 67.759 & 44.256 & 11.124 & 13.219 \\
\hline Umsatzänderg. 2006 ggü. 2005 & $+21 \%$ & $+12,1$ & $+5,5$ & $+22,8$ \\
\hline Gewinn vor Steuern 2006 in Mio. Euro & 5.133 & 3.657 & 1.230 & 1.183 \\
\hline Gewinn nach Steuern 2006 in Mio. Euro & 4.810 & 4.013 & 934 & 1.110 \\
\hline Eigenkapital in Mio. Euro & 47.845 & 14.111 & 7.091 & 4.401 \\
\hline Bilanzsumme in Mio. Euro & 127.232 & 93.455 & 18.725 & 28.094 \\
\hline Beschäftigte 2006 & 80.612 & 68.534 & 21.323 & 20.259 \\
\hline Angekündigte Investitionen & $\begin{array}{l}60 \text { Mrd. Euro bis } 2010 \\
\text { (inkl. Unternehmenskäufe) }\end{array}$ & 25 Mrd. Euro bis 2012 & $\begin{array}{l}6 \text { Mrd. Euro in Kraftwerke, } \\
\text { Netze + reg. Energien }\end{array}$ & $\begin{array}{l}\text { keine konkreten öffentlich } \\
\text { bekannten Planungen }\end{array}$ \\
\hline $\begin{array}{l}\text { Derzeitige (Haupt-)Gesellschafter } \\
\text { in \% }\end{array}$ & $\begin{array}{l}\text { Streubesitz } 87,34 \\
\text { Freistaat Bayern }(2) \\
\text { Allianz }(2,5), \text { UBS }(3,5)\end{array}$ & $\begin{array}{l}\text { Kommunen rund } 33 \\
\text { des Gesellschafterkapitals, } \\
\text { institutionelle Anleger }(37,9), \\
\text { Privatinvestoren }(13)\end{array}$ & $\begin{array}{l}\text { Vattenfall AB }(38,3) \text {, } \\
\text { Vattenfall Deutschland } \\
\text { GmbH }(58,11 \%) \text {, } \\
\text { Squeeze out-Verfahren }\end{array}$ & $\begin{array}{l}\text { ZV Oberschwäbische } \\
\text { Elektrizitätswerke }(45,01) \text {, } \\
\text { Electricite de France } \\
(E d F, 45,01)\end{array}$ \\
\hline Kraftwerkskapazitäten & $\begin{array}{l}\text { Erzeugungsportfolio deckt } \\
\text { alle Lastzeiten } a b\end{array}$ & $\begin{array}{l}\text { Erzeugungsportfolio deckt } \\
\text { alle Lastzeiten } a b\end{array}$ & $\begin{array}{l}\text { Erzeugungsportfolio deckt } \\
\text { alle Lastzeiten } a b\end{array}$ & $\begin{array}{l}\text { Erzeugungsportfolio deckt } \\
\text { alle Lastzeiten ab }\end{array}$ \\
\hline Erneuerbare Energie & $\begin{array}{l}\text { Mitte 2007: Aufbau Er- } \\
\text { neuerbare Energie und } \\
\text { Klimaschutzprojekte GmbH, } \\
\text { Investitionsplan: rd. } 6 \mathrm{Mrd} \text {. } \\
\text { Euro bis } 2010\end{array}$ & $\begin{array}{l}\text { Gründung RWE Innogy } \\
\text { GmbH am 01.02.2008; } \\
\text { geplantes Investitions- } \\
\text { volumen } 1 \text { Mrd. Euro p.a. }\end{array}$ & $\begin{array}{l}\text { Gründung Vattenfall } \\
\text { Europe New Energy GmbH } \\
\text { im Mai 2007; Investitions- } \\
\text { plan: ca. 1,5 Mrd. Euro } \\
\text { bis } 2013\end{array}$ & $\begin{array}{l}\text { Gründung der Renewables } \\
\text { GmbH im Frühsommer } \\
2007\end{array}$ \\
\hline
\end{tabular}

Stromerzeugung den bis dato selbst beherrschten Markt im Wettbewerbssinne zu öffnen. Dies wurde von der Monopolkommission (2004, S. 440ff.) mehrfach heftig kritisiert. Der deutsche „Sonderweg“ des „verhandelten Netzzugangs“ wurde schließlich auf massiven Druck der EU-Kommission durch die zweite Novellierung des EnWG im Jahr 2005 beendet, durch die eine staatliche Bundesnetzagentur für die Bereiche Strom und Gas eingerichtet wurde. Am 6. November 2007 trat dann die Verordnung über die Anreizregulierung der Energieversorgungsnetze (ARegV 2007) in Kraft. Diese Verordnung soll künftig der Bundesnetzagentur als weiteres Instrument zur Durchsetzung von Wettbewerb auf den Strom- und Gasmärkten dienen (Abschnitt 2).

\subsection{ENTWICKLUNG DER UNTERNEHMENSZAHL UND RECHTSFORMEN}

Bei den Elektrizitätsversorgungsunternehmen (EVUs) schrumpfte die Anzahl der Unternehmen nach der Liberalisierung zwischen 1998 und 2005 um rund $20 \%$ von 1.229 Gesellschaften mit dem Schwerpunkt Elektrizität auf nur noch 979, wobei mit 919 Unternehmen im Jahr 2001 der Tiefpunkt erreicht war. Dass in den Folgejahren wieder mehr Unternehmen am Markt präsent sind, ist unter anderem bedingt durch das "legal unbundling“, also die gesellschafts-, aber nicht eigentumsrechtliche Trennung der Netze von anderen Bereichen der integrierten Verbundunternehmen. Diese „Entflechtung“ ist seit der EnWG-Novellierung im Jahre 2005 für alle EVUs mit Ausnahme der ganz kleinen Betriebe zwingend vorgesehen.

Der „öffentliche Sektor" erlebte seit der Liberalisierung einen erheblichen Bedeutungsverlust. Dabei halbierte sich fast die Anzahl der EVUs in öffentlicher Rechtsform von $283 \mathrm{im} \mathrm{Jahr} 1998$ auf nur noch 153 Gesellschaften (2005). Ihr Anteil an den Unternehmen insgesamt ging von $23 \%$ auf $16 \%$ um jene sieben Prozentpunkte zurück, die im Gegenzug der Anteil der Betriebe in privater Rechtsform inklusive der verselbstständigten (privatisierten) Unternehmen in öffentlichem Besitz zulegte. Vor allem die Rechtsformen der $G m b H$ und Co. KG sowie der $G m b H$ verzeichneten dabei einen immensen Aufschwung. Die
Zahl der Aktiengesellschaften nahm demgegenüber vor allem aufgrund von Zusammenschlüssen größerer EVUs um 21 Unternehmen bzw. gut $17 \%$ ab.

\subsection{MEHR MARKTMACHT DURCH KONZENTRATION}

Der Rückzug des öffentlichen Sektors und Fusionen waren im Außenverhältnis die wesentlichen Antworten der EVUs auf die Marktöffnung. Unter dem Druck der neuen Marktgegebenheiten setzten gerade die vormals neun Verbundmonopolisten auf externe Unternehmenskonzentration und Beteiligungen an den Regionalversorgern sowie kommunalen Stadtwerken. Im Laufe der letzten zehn Jahre sind so aus den neun Unternehmen mit der E.ON AG, der RWE AG, der Vattenfall Europe AG sowie der EnBW Energie Baden-Württemberg AG vier führende Gesellschaften entstanden (Tabelle 1). Strategisch bereinigten diese „Big-4“ ihre Produktpalette in Richtung Kerngeschäfte und verkauften in großem Stil ihre energiefremden Sparten. Hinzu kam eine Internationalisierung.

Die augenblickliche Bilanzsumme dieser vier Unternehmen zusammen beträgt 


\begin{tabular}{|c|c|}
\hline \multicolumn{2}{|c|}{$\begin{array}{l}\text { Tabelle 2: Marktanteile am } \\
\text { Stromgroßkundenmarkt } 2004 \\
- \text { in \% - }\end{array}$} \\
\hline RWE & über 20 \\
\hline E.ON & über 15 \\
\hline EnBW & unter 15 \\
\hline Vattenfall Europe & deutlich unter 10 \\
\hline Händler & ca. 5 \\
\hline Stadtwerke & über 36 \\
\hline \multicolumn{2}{|c|}{$\begin{array}{l}\text { Quelle: Bundeskartellamt, Anlage A zum Schriftsatz B6- } \\
\text { 21/03 vom 30. November 2006, S. 13, zit. in Monopol- } \\
\text { kommission (2007, } \\
\text { S. 66). }\end{array}$} \\
\hline
\end{tabular}

rund 266 Mrd. €. Sie ist allerdings recht ungleich verteilt. Auf E.ON entfallen fast $48 \%$ und auf RWE $35 \%$ dieser Summe. EnBW liegt bei einem Anteil von knapp $11 \%$. Vattenfall kommt auf die restlichen $7 \%$. Vielfach wird daher in der Strombranche von einem marktbeherrschenden Dyopol von E.ON und RWE gesprochen. Bezogen auf den Strom-Großkundenmarkt (Tabelle 2) - lässt man Beteiligungen zunächst außen vor - liegen die einzelnen sowie die unterschiedlich kumulierten Marktanteile indessen unterhalb der kartellrechtlichen Eingreifkriterien des Gesetzes gegen Wettbewerbsbeschränkungen (GWB) (Tabelle 2 und 3).

Allerdings halten die vier Verbundmonopolisten über 282 Minder- und Mehrheitsbeteiligungen (Vollhals et al. 2007, S. 15) an regionalen und kommunalen Versorgern (Stadtwerke), sodass sie in der Elektrizitätserzeugung insgesamt im Laufe der Jahre immer mehr Marktmacht und „eine herausragende Stellung“ aufgebaut haben (Monopolkommission 2007a). Unter Berücksichtigung der Beteiligungen wurden 2004 - gemessen an den unterschiedlichen Konzentrationsgraden $\left(\mathrm{CR}_{\mathrm{i}}=\right.$ Marktanteile der i größten Unternehmen) in der Elektrizitätserzeugung - alle kartellrechtlichen Schwellenwerte im Hinblick auf eine marktbeherrschende Stellung nach $\S 19$ GWB überschritten (Tabelle 3).

Die „Big-4“ allein dominieren mittlerweile $80 \%$ des deutschen Elektrizitätserzeugungsmarktes. Bemerkenswert ist insbesondere, dass ihr Erzeugungsportfolio alle Lastzeiten abdeckt und sie seit Kurzem auch im Bereich der erneuerbaren Energien entsprechende Kapazitäten im großen Stil aufbauen bzw. aufkaufen. Bedenklich ist vor allem, dass die „Big-4“ in der Grundlast nahezu $100 \%$ sowie in der Spitzenlast etwa $85 \%$ des Marktes auf sich vereinen. Im Sinne wettbewerblicher Strukturen ist insbesondere die letzte Kennziffer vor dem Hintergrund der Besonderheiten des Strommarktes (Leitungsgebundenheit und Nichtspeicherbarkeit von Strom) als „,nicht unkritisch" anzusehen (Angenendt et al. 2007). Schließlich können zu Spitzenlastzeiten keine weiteren Erzeugungskapazitäten aktiviert werden, wodurch sich der Spielraum für kollusives Verhalten erhöht.

\subsection{RÜCKGANG DER BESCHÄFTI- GUNG}

Im Innenverhältnis ging der nach der Liberalisierung einsetzende Prozess der Neuausrichtung der EVUs eindeutig zulasten der Beschäftigten. So wurden massiv Arbeitsplätze abgebaut oder oftmals zu schlechteren Konditionen ausgelagert (Outsourcing). Von 1992 bis 2005 reduzierte

\begin{tabular}{|c|c|c|c|c|}
\hline$C R_{i}$ & 1995 & 2000 & 2004 & $\begin{array}{c}\text { GWB- } \\
\text { Schwellenwerte }\end{array}$ \\
\hline $\mathrm{CR}_{1}$ & 22,0 & 27,0 & 34,0 & 33,3 \\
\hline $\mathrm{CR}_{3}$ & 48,8 & 53,4 & 76,0 & 50,0 \\
\hline $\mathrm{CR}_{5}$ & 56,7 & 69,1 & 87,0 & 66,7 \\
\hline
\end{tabular}

Tabelle 4: Änderungsraten der Beschäftigung nach Größenklassen

\begin{tabular}{|c|c|c|c|c|c|}
\hline & \multirow{2}{*}{$\begin{array}{l}\text { Unternehmen mit } \\
\text {... Beschäftigten }\end{array}$} & \multirow{2}{*}{$\begin{array}{l}\text { Anzahl Unter- } \\
\text { nehmen } 2005\end{array}$} & \multirow{2}{*}{$\begin{array}{l}\text { Beschäftigte } \\
2005\end{array}$} & \multicolumn{2}{|c|}{$\varnothing$ Änderungsrate in \% } \\
\hline & & & & 1992-1997 & 1998-2005 \\
\hline \multirow{5}{*}{$\begin{array}{l}\text { Größen- } \\
\text { klassen }\end{array}$} & alle & 979 & 207.700 & $-2,4$ & $-2,7$ \\
\hline & $\geq 500$ & 84 & 147.148 & $-3,6$ & $-3,9$ \\
\hline & $250-499$ & 60 & 21.075 & k. A. & 0,1 \\
\hline & $100-249$ & 119 & 18.343 & k. A. & 0,5 \\
\hline & 50-99 & 179 & 12.847 & k. A. & 3,0 \\
\hline
\end{tabular}

sich die Zahl der sozialversicherungspflichtigen Beschäftigung in der Stromversorgung von 290.168 auf 207.654. ${ }^{3}$ Mithin gingen fast drei von zehn Arbeitsplätzen innerhalb von 13 Jahren in der Energiewirtschaft verloren! In der Phase seit der Marktöffnung waren davon rund 43.500 Mitarbeiter betroffen. Obwohl in Antizipation der Marktöffnung bereits vor 1998 Produktivität steigernde Maßnahmen eingeleitet und umgesetzt worden sind, hatte der zunächst intensivierte Wettbewerb - insbesondere in den ersten Jahren nach Inkrafttreten des neuen EnWG - einen beschleunigten Arbeitsplatzabbau zur Folge. Seit der Liberalisierung nahm die Beschäftigung jahresdurchschnittlich um 2,7 \% ab. Zuvor betrug die Rate noch 2,4 \% (Tabelle 4). Vorrangig traf es Unternehmen mit über 500 Beschäftigten. Bei den Stadtwerken mit bis zu 500 Beschäftigten ist demgegenüber von 1998 bis 2005 ein Zuwachs zu konstatieren.

\subsection{ANPASSUNGEN BEI DEN PERSONALKOSTEN}

Der Rückgang der Beschäftigung schlug sich insbesondere in den Arbeitskosten der Elektrizitätswirtschaft nieder. ${ }^{4}$ Von 1992 auf 1997 stiegen die Personalaufwendungen trotz des Beschäftigtenabbaus um über $7 \%$ an. Seitdem sind sie rückläufig. Von 1998 auf 2005 verringerten sich die Personalkosten um 2,5\% (Tabelle 5). In diesem Rückgang überlagern sich die gegenläufigen Wirkungen des Arbeitsplatzabbaus und einer Entgeltsteigerung: Denn seit der Marktöffnung erhöhten sich die Entgelte pro Kopf ebenso wie die pro Stunde um rund $18 \%$ bzw. jahresdurchschnittlich um 2,4 \%. Innerhalb der Entgeltstruktur wurde jedoch die Bedeutung der sonstigen Sozialkosten zurückgedrängt. Dieser Kostenblock beinhaltet im weitesten Sinne übertarifliche Lohnbestandteile, vor allem

\footnotetext{
3 Im Rahmen einer gesamtwirtschaftlichen Bilanz wären allerdings auch die indirekten Folgen des Erneuerbaren-Energien-Gesetzes (EEG) zu berücksichtigen. Nach Berechnungen der Bundesregierung sollen 2006 u.a. für die Erzeugung der alternativen Anlagen in anderen Wirtschaftsbereichen ca.124.000 Arbeitsplätze über das EEG geschaffen worden sein (Bundesministerium für Umwelt 2007, S. 5).

4 In der öffentlichen Statistik wurde die Erfassung der Personalkosten im Laufe der Zeitperiode verändert, sodass die Daten nur eingeschränkt vergleichbar sind.
} 


\begin{tabular}{|c|c|c|c|c|c|c|c|c|c|c|c|}
\hline & $\begin{array}{l}\text { alle Elektrizitäts- } \\
\text { unternehmen }\end{array}$ & 1998 & 1999 & 2000 & 2001 & 2002 & 2003 & 2004 & 2005 & $\begin{array}{c}1998-2005 \\
\text { in \% }\end{array}$ & $\begin{array}{l}\varnothing \text { Änderg. } \\
\text { p.a. in \% }\end{array}$ \\
\hline \multirow{6}{*}{ 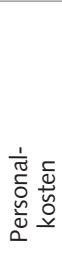 } & Beschäftigte & 251.297 & 239.777 & 219.586 & 205.816 & 207.419 & 198.758 & 209.667 & 207.654 & $-17,4$ & $-2,7$ \\
\hline & Arbeitsstd. in Tsd. & 389.726 & 371.287 & 343.021 & 314.633 & 313.351 & 303.929 & 323.137 & 321.198 & $-17,6$ & $-2,7$ \\
\hline & Arbeitsstd. je Mitarbeiter & 1.551 & 1.548 & 1.562 & 1.529 & 1.511 & 1.529 & 1.541 & 1.547 & $-0,3$ & $-0,0$ \\
\hline & ... in Mio. Euro & 14.136 & 14.179 & 14.050 & 12.329 & 12.450 & 12.833 & 13.264 & 13.777 & $-2,5$ & $-0,4$ \\
\hline & ... je Beschäftigten in Euro & 56.251 & 59.134 & 63.984 & 59.903 & 60.023 & 64.566 & 63.262 & 66.346 & 17,9 & 2,4 \\
\hline & ... je Arbeitsstd. in Euro & 36,27 & 38,19 & 40,96 & 39,19 & 39,73 & 42,22 & 41,05 & 42,89 & 18,3 & 2,4 \\
\hline \multirow{3}{*}{ 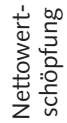 } & .... vor Steuern in Mio. Euro & 25.306 & 27.536 & 22.629 & 21.900 & 24.442 & 23.729 & 29.350 & 31.388 & 24,0 & 3,1 \\
\hline & ... je Beschäftigten in Euro & 100.703 & 114.840 & 103.053 & 106.406 & 117.839 & 119.386 & 139.984 & 151.155 & 50,1 & 6,0 \\
\hline & ... je Arbeitsstd. in Euro & 64,93 & 74,16 & 65,97 & 69,60 & 78,00 & 78,07 & 90,83 & 97,72 & 50,5 & 6,0 \\
\hline
\end{tabular}

\begin{tabular}{|c|c|c|c|c|c|c|c|c|c|c|}
\hline $\begin{array}{l}\text { alle Elektrizitäts- } \\
\text { unternehmen } \\
\text { (Angaben in Mio. Euro) }\end{array}$ & 1998 & 1999 & 2000 & 2001 & 2002 & 2003 & 2004 & 2005 & $\begin{array}{c}1998-2005 \\
\text { in } \%\end{array}$ & $\begin{array}{l}\varnothing \text { Änderg. } \\
\text { p.a. in \% }\end{array}$ \\
\hline Lohn und Gehalt & 14.136 & 14.179 & 14.050 & 12.329 & 12.450 & 12.833 & 13.264 & 13.777 & $-2,5$ & $-0,4$ \\
\hline Fremdkapitalzinsen & 1.469 & 1.636 & 1.275 & 1.326 & 1.238 & 1.187 & 1.128 & 1.137 & $-22,6$ & $-3,6$ \\
\hline Mieten und Pachten & 920 & 967 & 817 & 1.472 & 1.136 & 1.294 & 1.499 & 2.288 & 148,7 & 13,9 \\
\hline Steuern und Abgaben & 4.340 & 4.768 & 4.181 & 4.130 & 4.480 & 4.549 & 4.979 & 5.094 & 17,4 & 2,3 \\
\hline Gewinne nach Ertragssteuern & 4.442 & 5.986 & 2.306 & 2.643 & 5.138 & 3.866 & 8.480 & 9.092 & 104,7 & 10,8 \\
\hline Nettowertschöpfung gesamt & 25.306 & 27.536 & 22.629 & 21.900 & 24.442 & 23.729 & 29.350 & 31.388 & 24,0 & 3,1 \\
\hline
\end{tabular}

Aufwendungen für die betriebliche Altersversorgung. Der Anteil der sonstigen Sozialkosten an den gesamten Personalkosten nahm von knapp 17 \% im Jahre 1998 auf $14,5 \%$ im Jahre 2005 ab. Wird das Jahr 2000 als Basis genommen, als der Anteil der sonstigen Sozialkosten an den Personalkosten mit 19,4 \% am höchsten lag, betrug der Abbau innerhalb der folgenden fünf Jahre sogar fünf Prozentpunkte!

\subsection{PROGRESSIVE EINKOMMENSUMVERTEILUNG}

Parallel zu den Personalkosten erhöhten sich im betrachteten Zeitabschnitt (abgesehen von 1999) auch die jährlichen Umsätze der EVUs. Allerdings beinhaltet diese Kennzahl Vorleistungen und Abschreibungen, welche den Umsatz als Leistungsmaß der Unternehmen erheblich verzerren; in der Stromwirtschaft gilt dies insbesondere im Falle eines Anstiegs der Primärenergiepreise. Den eigentlichen wirtschaftlichen „Veredelungsprozess“ eines Unternehmens gibt daher der Indikator der Nettowertschöpfung an, der nach einem zwischenzeitlichen Rückgang über den Beobachtungszeitraum hinweg um $24 \%$ bzw. jahresdurchschnittlich um 3,1 \% zugelegt hat. Die Höhe der Nettowertschöpfung einer Periode kann im Rahmen der Entste- hungsrechnung einerseits aus dem am Umsatz anknüpfenden Produktionswert ${ }^{5}$ vermindert um die Vorleistungen und die Abschreibungen - bestimmt werden. Diese Wertschöpfung kann andererseits komplett für Personal-, Zins-, Miet- und Pachtaufwand, Steuern und Abgaben (hier insbesondere Konzessionsabgaben) sowie den Bilanzgewinn der jeweiligen Periode verwendet werden.

Aus der Relation zwischen Wertschöpfung und geleisteten Arbeitsstunden ergibt sich die Arbeitsproduktivität. Hier kam es von 1998 bis 2005 zu einem Anstieg um 50,5 \% bzw. einem jahresdurchschnittlichen Zuwachs von $6 \%$ (Tabelle 5). Vor allem in den Jahren 2004 und 2005 ist ein erheblicher Schub zu verzeichnen. Da aber die Einkommen je Arbeitnehmerstunde mit 2,4\% p.a. deutlich langsamer als die Arbeitsproduktivität anstiegen, müssen folglich die Einkommen anderer Gruppen im betrachteten Zeitraum stärker zugelegt haben. Und tatsächlich: Insbesondere die Einkommenspositionen $\mathrm{Ge}$ winne sowie Mieten und Pachten zeichneten sich dabei durch überdurchschnittliche Zuwächse aus (Tabelle 6). Von der Öffnung der Strommärkte profitierte die „Kapitalseite“ demnach progressiv. Auffällig waren im Einzelnen die folgenden Entwicklungen:
(1) Sowohl die Lohn- und Gehaltssumme als auch die Fremdkapitalzinsen verzeichnen seit 1998 Rückgänge. Der Anteil des Personalaufwands an der Wertschöpfung fiel im betrachteten Zeitraum extrem von knapp $56 \%$ auf rund $44 \%$. Hintergrund für die rückläufigen Zinsaufwendungen sind verstärkte Innenfinanzierungsmöglichkeiten, rückläufige Zinssätze sowie eine gesunkene Inanspruchnahme von Krediten wegen nachlassender Investitionstätigkeit der EVUs (vgl. Abschnitt 1.6).

(2) Die staatliche Seite als Wertschöpfungsempfänger konnte die Einkünfte mittels Konzessionsabgaben, Verbrauchs- und Ertragssteuern von 4,3 Mrd. $€$ in 1998 auf 5,1 Mrd. $€$ in 2005 um 18,6\%, also jahresdurchschnittlich um 2,3\%, erhöhen.

(3) Demgegenüber wuchsen die Gewinne um ca. $105 \%$ bzw. durchschnittlich um fast $11 \%$ p.a., während die allerdings relativ weniger bedeutenden Aufwendungen für Mieten und Pachten in der Referenzperiode sogar um fast $150 \%$ bzw. jahres-

\footnotetext{
5 Dieser entspricht weitgehend den Umsätzen, da Bestands- bzw. Lagerveränderungen wegen der Nichtspeicherbarkeit von Strom irrelevant und aktivierte Eigenleistungen vernachlässigbar sind.
} 
Tabelle 7: Investitionen nach Größenklassen

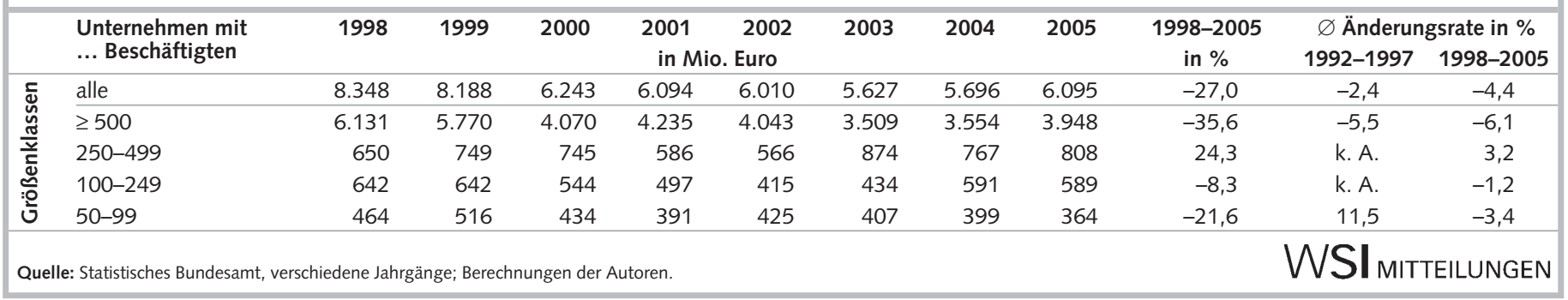

durchschnittlich um beinahe $14 \%$ zulegten. Betrug der Anteil der Gewinne 1998 an der Wertschöpfung noch $17,5 \%$, so waren es 2005 bereits fast $29 \%$.

\subsection{INVESTITIONSATTENTISMUS}

Die hohen Unternehmensgewinne basieren nicht nur auf Kürzungen der Personalkosten, sondern zu einem großen Teil auch auf Erträgen aus Investitionen der Vergangenheit und der seit der Liberalisierung beobachtbaren Zurückhaltung bei Modernisierungen - sowohl im Kraftwerkspark als auch bei den Netzen. Die hierfür eingesetzten Mittel sanken von 1998 auf 2005 um 27 \% bzw. 4,4 \% p.a. und damit viel dynamischer als in der Vorperiode (Tabelle 7). Investitionsattentismus, also eine abwartende Haltung, zeichnete insbesondere die großen Energieversorger mit jahresdurch- schnittlichen Rückführungen von über $6 \%$ aus. Vor diesem Hintergrund überrascht es nicht, dass die Bundesnetzagentur jüngst erheblichen Ausbaubedarf bei den Stromübertragungsnetzen sieht (BNetzA 2008). Hinzuzufügen ist, dass die Erneuerung des Kraftwerksparks ebenfalls langsamer voranschreitet als geplant (bdew 2008).

\section{Wettbewerb und Preisentwicklung}

\subsection{ENTTÄUSCHTE ERWARTUNGEN}

Gemessen an den vereinzelt hochgesteckten Erwartungen ${ }^{6}$ zur Preisentwicklung wirken hierzulande die tatsächlichen Erfolge der Liberalisierung in einer längerfristigen Betrachtung eher ernüchternd.
Abb. 1: Durchschnittlicher Strompreis, Drei-Personen-Haushalt mit Jahresverbrauch von 3.500 kWH/a* - inkl. Stromsteuer, in ct/kWH -

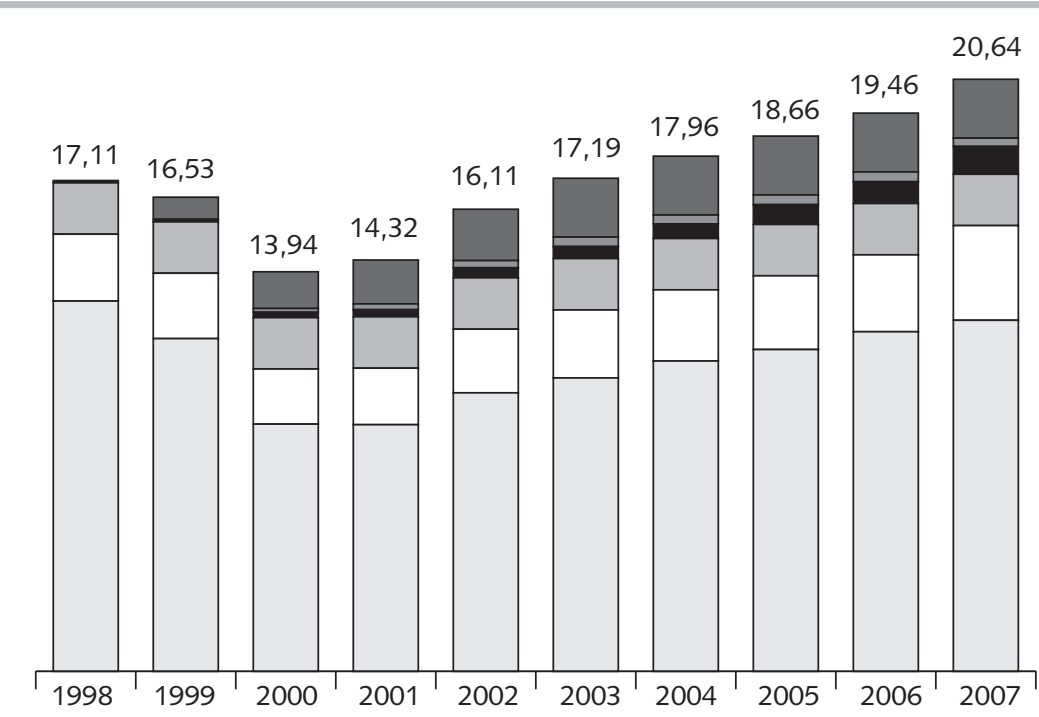

$\square$ Erzeugung $\square$ Mehrwertsteuer $\square$ Umlage nach Kraft-Wärme-Kopplungsgesetz Umlage nach Erneuerbare-Energien-Gesetz $\square$ Konzessionsabgabe $\square$ Stromsteuer

*Gesamtbelastung durch KWKG ab 2002 gesunken; durch Entlastung Industrie steigende Belastung für Haushalte. Quelle: Bundeswirtschaftsministerium (2008, S. 25).
Deutschland zählt im EU-Vergleich immer noch zu den Ländern mit den höchsten Strompreisen. Zwar kam es unmittelbar nach der Marktöffnung - gegenüber 1998 und unter Herausrechnen von staatlich veranlassten Preisbestandteilen - in der Spitze zu bemerkenswerten Nachlässen von $41 \%$ beim Industriestrom (ohne Abbildung) bzw. $33 \%$ beim Haushaltsstrom (Abbildung 1). Sie erwiesen sich indessen als flüchtig, denn inzwischen sind diese Preisvorteile - anders als etwa im Bereich der ebenfalls liberalisierten Telekommunikation - weitgehend wieder aufgezehrt. Ohne die administrierten Komponenten lagen die Entgelte für die privaten Haushalte zuletzt nur noch um gut $4,5 \%$ und für die Industrie um knapp $3 \%$ niedriger, und dies bei einem Produktivitätsfortschritt - schreibt man die oben berechnete Dynamik von jährlich $6 \%$ bis 2007 fort von fast $70 \%$.

Angesichts der zuvor beschriebenen Markt- und vertikalen Verbundstrukturen ist dieses Ergebnis nicht überraschend. Durch die Konzentration ist zum einen der Spielraum für kollusives oder gar missbräuchliches Marktverhalten der EVUs immens (Schmidt 2005, S. 175f.). Mit Blick auf die Strompreiserhöhungen Ende 2007 glaubte der Präsident des Bundeskartellamtes sogar, seine Behörde habe „starke Indizien " für Preisabsprachen gefunden (Handelsblatt 8.11.2007). Zum anderen verhinderte die lange Zeit faktisch unbeeinträchtigte Netzhoheit der Verbundunternehmen über prohibitiv hohe Durch-

6 Die Erwartungen bei Verabschiedung des EnWG im Jahr 1998 waren genau genommen gespalten. Der damalige Bundeswirtschaftsminister Rexrodt gab sich überzeugt, das neue Recht werde "zu wettbewerbsfähigen Strom- und Gaspreisen beitragen, von denen alle Verbraucher profitieren werden" (Handelsblatt 28.4.1998). Auch die ÖTV sah in dem Gesetz verbesserte Wettbewerbsstrukturen (vgl. Handelsblatt 2.3.1998). Andere Stimmen, wie die des Veba-Chefs Hartmann, waren verhaltener (vgl. Wirtschaftswoche 12.3.1998). 


\section{Tabelle 8: Wertschöpfungsrech- nung im Netzbetrieb - in \% des} Umsatzes - *

\begin{tabular}{lr}
\hline Umsatz & 100,0 \\
- Vorleistungen u. Abschreibungen & 56,5 \\
\hline = Nettowertschöpfung & 43,5 \\
\hline \multicolumn{2}{|c|}{ davon: } \\
$\quad$ Personalaufwand & 24,2 \\
+ Zinsertrag & 3,4 \\
+ Steuern & 2,8 \\
+ Gewinn & 13,1 \\
\hline
\end{tabular}

* Der Miet- und Pachtaufwand ist nicht ausgewiesen. Dieser ist in den Vorleistungen inkl. der Abschreibungen enthatten, sodass die Wertschopfungsquote um diese Größe zu schen Bundesbank gilt dies aber auch für die Daten und Be chnungen bezogen auf die Gesamtwitschaft. rechnungen bezogen auf die Gesamtwirtschaft.

Quelle: Berechnungen der Autoren nach Daten von Diekmann et a. leitungsentgelte das Entstehen eines nachhaltigen Wettbewerbs unter den Stromanbietern. In der Tat lassen hier die wirtschaftlichen Daten der Netzbetreiber auf überhöhte Entgelte schließen. So konnten einzelne EVUs im Netzbetrieb im Jahr 2005 eine überdurchschnittlich hohe Umsatzrendite von 13,1\% und einen ebenso überragenden Wertschöpfungsanteil am Umsatz von 43,5\% vorweisen (Tabelle 8). Zum Vergleich: Über die Gesamtwirtschaft hinweg belief sich die Umsatzrendite in 2005 lediglich auf 3,4\% und die Wertschöpfungsquote auf 23,1 \% (Deutsche Bundesbank 2007, S. 39).

\subsection{NEUE WETTBEWERBSIMPULSE}

Vor diesem Hintergrund und in Anbetracht der „Rekordgewinne“ (insbesondere der „Big-4“) bei gleichzeitig wieder steigenden Strompreisen kann auch die derzeit geführte Entflechtungsdebatte (von Hirschhausen et al. 2007 (pro); Ockenfels 2007 (kontra)) bezogen auf die „Big-4“ nicht überraschen. Im Gegenteil: Selbst die EUKommission zeigt sich von den hiesigen Ergebnissen der von ihr initiierten Marktöffnung enttäuscht und hat dieser Debatte mit einem erneuten Vorstoß zur eigentumsrechtlichen Zerschlagung („Ownership Unbundling“) eine neue Dimension verliehen. Kommissionspräsident Barroso erklärte die Zeit geduldiger Trippelschritte für beendet und begründete seine Initiative mit den Worten: „Wenn Unternehmen Strom $[\ldots]$ verkaufen und gleichzeitig die Netze kontrollieren, dann haben sie allen Anreiz, Konkurrenten den fairen Zugang zu ihren Netzen zu verweigern" (Handelsblatt 20.9.2007).
Auch die Bundesregierung sieht die Notwendigkeit zur Nachsteuerung, denn die ,jüngsten Strom-[...]preiserhöhungen zeigen, dass es mehr Wettbewerbs im Energiebereich bedarf" (Bundeswirtschaftsministerium 2008, S. 24). Dabei würde ihr zunächst eine Verschärfung der Legal-Unbundling-Vorschriften als "dritter Weg“ ausreichen. Zudem stützen sich die Maßnahmen neben der Erleichterung des Anbieterwechsels für Tarifkunden durch die seit 2007 wirksame Stromgrundversorgungsverordnung im Wesentlichen auf das Kartellrecht und eine Anreizregulierung für die Netzentgelte.

\section{GWB-NOVELLE UND ...}

Mit Wirkung vom 1. Januar 2008 wurde das Gesetz gegen Wettbewerbsbeschränkungen (GWB) durch die bis 2012 befristete Einführung des neuen $\$ 29$ novelliert. Zielsetzung ist es, die Anwendung des Missbrauchstatbestandes im $\S 19$ GWB auf die Energiewirtschaft an Effektivität zu schärfen und die aufgrund des fehlenden Wettbewerbs durch den Markt nicht abgebauten Monopolgewinne notfalls durch den Staat abzuschmelzen. ${ }^{7}$

Nach dieser neuen Vorschrift ist es Elektrizitätsanbietern verboten, eine alleinige oder zusammen mit anderen ausgeübte marktbeherrschende Stellung (festzustellen anhand der Schwellenwerte des $\S 19$ GWB) missbräuchlich auszunutzen:

(1) Als Missbrauch zählt - im Sinne eines Vergleichs(-markt)konzeptes, - in Relation zu anderen Versorgungsunternehmen oder zu Anbietern auf vergleichbaren Märkten ungünstigere Entgelte bzw. Geschäftsbedingungen zu verlangen, es sei denn, dies lasse sich sachlich rechtfertigen. Dabei wird beim Vergleich mit anderen Versorgungsunternehmen zugleich die Beweislast umgekehrt. Die Kartellbehörden sind nämlich jetzt „befugt, einen Missbrauchsverdacht auszusprechen, sobald ein marktbeherrschendes Versorgungsunternehmen sein Entgelt [erheblich] über den Preis eines beliebigen anderen Versorgungsunternehmens anhebt, ohne - wie bisher [...] üblich - die strukturelle Vergleichbarkeit nachweisen zu müssen" (Monopolkommission 2007b, S. 4). Es obliegt jetzt im Fall von beanstandeten Preisunterschieden dem Unternehmen, die sachliche Begründung gegenüber den Kartellbehörden aufzuklären.
(2) Als Missbrauch gilt auch - im Sinne eines Gewinnbegrenzungsansatzes - ein unangemessenes Überschreiten der Kosten durch die Entgeltpreise. Ausgenommen von diesen Regelungen bleibt der Netzbetrieb.

\section{... ANREIZREGULIERUNG}

Hinsichtlich des Netzbetriebs sind die Trennungsvorschläge der EU-Kommission heftig umstritten. Fraglich ist beispielsweise, ob sich eine Entflechtung angesichts der erwartbaren Verluste bei den Economies of Scale aus ökonomischer Sicht am Ende überhaupt für die Gesellschaft lohne bzw. ob es nicht effizientere Maßnahmen gibt, um den Wettbewerb wirkungsvoll zu beleben (Monopolkommission 2007a). Problematisch wäre es auch, wenn in der Energieversorgung nach einer Zerschlagung unter Umständen zwei Sparten mit Machtkonzentration entstehen. Überdies wären langwierige rechtliche Auseinandersetzungen mit unbestimmtem Ausgang zu befürchten. Mit Blick auf den Standort kommt hinzu, dass Deutschland bei der Erfüllung der Kommissions-Forderungen dann gegebenenfalls in der EU ziemlich allein dastehen könnte, wenn in einzelnen Nachbarländern eine Zerschlagung in noch staatlichen Unternehmen unterbleibt und Ausnahmeregeln für EU-Randländer getroffen werden.

Vor diesem Hintergrund plädieren das Bundeswirtschaftsministerium (2008) und auch die Monopolkommission (2007a) dafür, das Legal-Unbundling zu verschärfen und zunächst einmal die Auswirkungen der am 6. November 2007 in Kraft getretenen Verordnung über die Anreizregulierung der Energieversorgungsnetze abzuwarten. Sie soll insbesondere $\mathrm{zu}$ angemessenen Durchleitungspreisen führen und damit in Verbindung mit der seit Mitte 2007 gültigen Kraftwerknetzanschlussverordnung einen wettbewerbsfähigen, diskriminierungsfreien Zugang von Mitanbietern der Stromerzeugung zu den ehemaligen „Besatzungszonen" eines Verbundmonopolisten ermöglichen.

Das derzeit noch geltende System der „detaillierten Kostenprüfung“ wird zum

\footnotetext{
7 In einer Anhörung zur GWB-Novelle des Ausschusses für Wirtschaft und Technologie im Deutschen Bundestag wurde festgestellt, dass es , ,erhebliche Monopolaufschläge' von bis zu 9,5 Mrd. Euro gebe, die den Verbrauchern , aus der Tasche' gezogen würden“ (Deutscher Bundestag 2007).
} 
1. Januar 2009 durch die anreizbasierte Regulierung abgelöst (ARegV 2007). Der Fokus des neuen Regulierungsrahmens liegt sowohl auf generellen Kostensenkungen als auch auf Effizienzsteigerungen. Vor Beginn der Regulierungsperiode werden die zulässigen Preise für jedes Jahr des mehrperiodigen Regulierungszeitraums festgelegt. Die Preisobergrenzen werden innerhalb dieser Phase jeweils zum 1. Januar eines Jahres angepasst, wenn sich entweder der Verbraucherpreisindex oder der Anteil nicht beeinflussbarer Kosten ändert.

Netzbetreiber werden in den nächsten Jahren ihre strategischen und operativen Entscheidungen auf dieser Grundlage zu treffen haben. Die ARegV verpflichtet die Betreiber, den Teil der Kosten jährlich zu senken, den sie „beeinflussen “können. Das Ausgangsniveau der Kostenminderungen für den ersten fünfjährigen Regulierungszeitraum ist nach $\$ 6(2) \mathrm{ARegV}$,das Ergebnis der Kostenprüfung der letzten Genehmigung der Netzentgelte nach $\$ 23$ a des Energiewirtschaftsgesetzes vor Beginn der Anreizregulierung", wobei derzeit in der Diskussion ist, dieses auf das Jahr 2006 zu fixieren. Die Reduktionsrate setzt sich dabei aus einem allgemeinen (a) und einem vom jeweiligen Netzbetreiber abhängigen Teil (b) zusammen:

a) Der sektorale Produktivitätsfaktor, den alle Netzbetreiber erfüllen müssen, wird ab Anfang 2009 ausgehend von den 2006er Werten - für die ersten fünf Jahre mit jährlich 1,25\% angesetzt. In der anschließenden Fünfjahresperiode ab Anfang 2014 beläuft er sich auf $1,5 \%$ p.a. (vgl. $\$ 9$ ARegV). Bis Ende 2018 ist folglich eine Minderung um gut $18 \%$ vorgesehen.

b) Zudem führt die Bundesnetzagentur einen bundesweiten Effizienzvergleich mittels Aufwands- und Vergleichsparametern durch (Benchmark auf der Basis der $\$ \$ 12$ bis $15 \mathrm{ARegV}$ ). Ziel ist es, Ineffizienzen eines individuellen Netzbetreibers zu ermitteln und diese im Vergleich mit dem kostengünstigsten Akteur im Rahmen des derzeitigen Regulierungszeitraums abzubauen.

Mit der Festlegung eines zehnjährigen Rahmens ist der Gesetzgeber Befürchtungen u.a. vom Verband kommunaler Unternehmen (VKU) - entgegengekommen und hat eine ausreichende Zeitspanne eingeräumt, um das Kostenniveau vergleichbarer Unternehmen erreichen zu können (VKU
2006). Das Verfahren berücksichtigt zugleich die Struktur der Netzbetreiber in Deutschland, die im Vergleich zu anderen Staaten durch Vielfalt und Heterogenität geprägt ist. Gemäß $\$ 16(2) \mathrm{ARegV}$ kann die Regulierungsbehörde zudem Netzbetreibern abweichende Effizienzparameter vorgeben, wenn diese Betriebe nachweisen können, dass die für sie geltenden Effizienzvorgaben unter Nutzung aller möglichen und zumutbaren Maßnahmen nicht erreicht bzw. übertroffen werden können. Unzumutbar sind in diesem Kontext auch Maßnahmen, die „ein Unterschreiten von Arbeitsbedingungen zur Folge haben, die in dem nach dem Energiewirtschaftsgesetz regulierten Bereich üblich sind“.

Zentral für die Ausgestaltung des Regulierungsrahmens ist die sogenannte Beeinflussbarkeit von Kosten, weil nur beeinflussbare Kosten dem Anpassungsdruck unterliegen. Die dauerhaft nicht beeinflussbaren Kosten und deshalb von Minderungen ausgenommen Kosten sind explizit in $\$ 11$ ARegV festgelegt. Als zumindest vorübergehend nicht beeinflussbare Kosten gelten die Kostenanteile, die entweder effizient sind oder auf strukturellen Besonderheiten beruhen. Zudem ist bis Ende 2008 ein „Moratorium" für Arbeitskosten vereinbart. Danach aber stehen trotz grundgesetzlich geschützter Tarifautonomie Sonderzahlungen, Urlaubsvergütungen, betriebliche Altersversorgung, Altersteilzeit, Lohnfortzahlung im Krankheitsfall sowie Ausgaben für die Ausbildung auf dem Prüfstand der Bundesnetzagentur. Sie gelten dann als beeinflussbare Kosten ( $\$ 11(4) \mathrm{ARegV})$ und unterliegen dem Anpassungsdruck.

\section{Entwicklung der Verteilungskonflikte}

\subsection{IM DREIECK KUNDEN, BESCHÄFTIGTE, UNTERNEHMEN}

Eingangs konnte herausgearbeitet werden, dass die Elektrizitätswirtschaft mit ihren Kernsparten Erzeugung, Netzbetrieb, Handel und Vertrieb seit der Liberalisierung ihre Produktivität merklich erhöht hat. Obwohl keine detaillierten öffentlichen Zahlen vorliegen, zeigen erste Befragungen bei Stadtwerken (vgl. Fußnote 1), dass die Produktivitätszuwächse bei den Netzbetreibergesellschaften allein mindestens gleich hoch ausfielen. Diese Effizienzfortschritte schlagen sich aber nur unzureichend in den Netzentgelten bzw. Strompreisen nieder (Leprich 2007, S. 21ff.), sodass am Ende die Stromkunden nicht im erhofften Umfang als Verteilungsgewinner aus der Liberalisierung hervorgegangen sind. Stattdessen konnten über die ganze Branche hinweg trotz rückläufiger Beschäftigung spürbare Umsatzzuwächse verbucht werden. Damit war nach der Bereinigung um Vorleistungen und Abschreibungen ein deutliches Anziehen der Nettowertschöpfung zu konstatieren. Nach Abzug der weitgehend durch langfristige Kontrakte bestimmten Zins-, Miet- und Pachtaufwendungen sowie der staatlichen Abgaben war der in den EVUs zur Verteilung stehende „Kuchen“ größer als zuvor. Den mit Abstand größten Teil des Zuwachses verleibten sich die Kapitaleigentümer ein, während die Arbeitnehmer kaum partizipierten. Ihnen drohte eher die Gefahr des Beschäftigungsabbaus. Doch immerhin hat die gewachsene Verteilungsmasse den Verteilungskonflikt innerhalb der Unternehmen bislang noch abfedern können.

Nun aber verliert die Politik zunehmend die Geduld, zumal sich einer Allensbach-Umfrage zufolge $92 \%$ der Deutschen von den Strom- und Gasversorgern „regelrecht abgezockt fühlen“ (Handelsblatt 6.1.2008). Die Apologeten des Marktes und Befürworter der von der Wirtschaft selbst gestalteten Rahmenbedingungen (Verbändevereinbarungen) erkennen immer mehr, dass angesichts der vorherrschenden Strukturen die angestrebte Umverteilung zugunsten der Kunden ohne ein Mehr an staatlichem Druck bzw. staatlicher Regulierung ausbleibt. Die Kombination aus nochmals vereinfachtem Anbieterwechsel, einer verschärften Missbrauchsaufsicht und der Anreizregulierung dürften den EVUs immer mehr zusetzen. Zwar ist umstritten, ob die Verschärfung des GWB nicht gar kontraproduktiv wirken könnte. ${ }^{8}$ Den-

\footnotetext{
8 So die Monopolkommission (2007b), die auf Basis des Entwurfs zu bedenken gab, das Gesetz sei primär symptom- und nicht ursachenbezogen, es nehme geradezu den Anreiz zu Preissenkungen, da man als Vorreiter bei Preissenkungen wisse, die Branche werde nachziehen müssen, während die Umsetzung der Gewinnbegrenzung erhebliche Schwierigkeiten bereite, die Kosten angemessen zu ermitteln. Die Endfassung des Gesetzes hat den Erheblichkeitszuschlag jedoch beibehalten, sodass nunmehr nicht jede Preissenkung auch von den Wettbewerbern nachvollzogen werden muss.
} 
noch scheint insbesondere die Anreizregulierung geeignet $\mathrm{zu}$ sein, weitere Produktivitätspotenziale zu entfalten, die Märkte effektiv zu öffnen und dadurch zusätzliche Preissenkungen zu erschließen. Schon jetzt, bevor die Regulierung richtig greift, weist das Bundeswirtschaftsministerium (2008, S. 25) darauf hin, dass die Netzentgelte im Genehmigungsverfahren um bis zu $20 \%$ abgesenkt wurden. Sollte das Maßnahmenpaket nicht reichen, mahnt das Bundeswirtschaftsministerium (2008, S. 29) vorsorglich: „Es ist [...] nicht auszuschließen, dass weitere Maßnahmen zur Nachsteuerung notwendig werden, damit der Wettbewerb auf den Strom-[...]märkten die gewünschte Dynamik entfaltet." In Wartestellung steht zudem der Vorschlag der Monopolkommission (2007a), beim Kraftwerksbau ein Moratorium in Bezug auf die Großkonzerne durchzuführen, um die Zahl der Erzeuger zu erhöhen. Über allem schwebt dann noch die Drohung des „Ownership-Unbundling" der EU-Kommission. Gegenwärtig versucht die deutsche Politik, deren Maßnahmen abzuwehren. Allerdings scheint die Androhung einer EURegelung allein auszureichen, um die EVUs gegenüber verschärften nationalen Regeln konzessionsbereiter zu machen.

Die Sparten der EVUs stehen damit aber zunehmend und in unterschiedlicher Form unter Druck, erzielte und zukünftige Produktivitätsfortschritte auch durch Preisnachlässe an die Kunden weiterzureichen und deren Verteilungsposition zu stärken. ${ }^{9}$ Dies dürfte der unternehmensinternen Verteilungsauseinandersetzung eine vollkommen neue Dimension verschaffen.

\subsection{AKZENTUIERUNG INTERNER VERTEILUNGSKONFLIKTE}

In der Erzeugungs-, Handels- und Vertriebssparte ist - wenn die politischen Instrumente greifen - mit einer stark abgeflachten Preisdynamik zu rechnen. Greifen die Maßnahmen nicht, werden sie weiter verschärft. Bei relativ preisunelastischer Nachfrage wird dies zu Umsatzeinbußen der Branche führen, was letztlich eine verringerte Wertschöpfung und damit erstmals eine nachhaltig kleinere Verteilungsmasse in den EVUs bedeuten würde. Hinzu kommt, dass angesichts des Investitionsstaus im Kraftwerksbau und der zukünftig knapperen und dann auch nicht mehr vollständig kostenfreien $\mathrm{CO}_{2}$-Zertifikate-Zuteilung auf längere Sicht der unterneh- mensinterne „Verteilungskuchen“ noch kleiner wird. Hinsichtlich der unternehmensinternen Verteilung aber zu glauben, eine Begünstigung der Stromabnehmer würde automatisch zulasten der Kapitaleigner gehen und dort die Monopolrenten abschöpfen, ist, um es vorsichtig auszudrücken, eher unwahrscheinlich und widerspricht auch den Erfahrungen der bisherigen Verteilungsauseinandersetzung (vgl. Abschnitt 2). Im Gegenteil, die Kapitaleigentümer scheinen den Gewinn nicht als Restgröße anzusehen, sondern sich daran gewöhnt zu haben, dass ihnen eine bestimmte Rendite zusteht. Das bedeutet aber letztlich, unter dem Druck von Beschäftigtenabbau die Löhne und Gehälter, die in marktwirtschaftlichen Ordnungen eigentlich kontraktbestimmte Größen sind, zur Residualgröße abzustempeln. Mit anderen Worten: Die Beschäftigten müssen sich dauerhaft darauf einstellen, die steigenden Anpassungslasten zu tragen. Dabei droht zudem eine eigendynamische Zuspitzung des Distributionskampfes. Kommt es zu produktivitätssteigernden Einsparungen beim Personal, steigt der Druck, diesen Kostenvorteil an die Kunden weiterzugeben.

Ähnlich, wenngleich nicht identisch, sieht es in der Netzsparte aus. Als Folge der Anreizregulierung sind die Netzentgelte bereits drastisch gesunken; weitere, eher verschärfte Runden stehen noch bevor. Mit Blick auf die Verteilungsmasse in der Branche bedeutet dies, dass bei unveränderter Durchleitungsmenge ${ }^{10}$ die Umsätze schrumpfen und damit ceteris paribus die Wertschöpfung zurückgeht. Erschwerend für die interne Distribution kommt - selbst wenn die Anreizregulierung dafür Abwälzungsspielräume lässt hier möglicherweise hinzu, dass sich angesichts der investiven Zurückhaltung ein enormer Engpass in der Netzinfrastruktur abzeichnet. Er muss in den nächsten Jahren beseitigt werden, um die Versorgungssicherheit gewährleisten zu können - zumal bei zunehmender Integration regenerativer Energien Strom über immer weitere Entfernungen transportiert werden muss. Somit steht der Netzsparte bei sinkenden Entgelten eine drastische, vielleicht sogar noch viel intensivere Verschärfung der unternehmensinternen Verteilungsauseinandersetzung als in der Erzeugungssparte bevor. Damit einher ginge auch die Gefahr der tarifpolitischen Abkoppelung beider Sparten. Dabei glaubt auch ver.di nicht, dass die geplanten Reduktionen der Netz- entgelte die Gewinne der Unternehmen insgesamt angreifen werden. Ver.di (2008) sieht eher die Eigenkapitalsubstanz einzelner Unternehmen und bis zu 40.000 Arbeitsplätze in den nächsten zehn Jahren gefährdet.

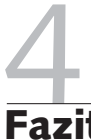

Fazit

Alles in allem droht somit ein pervertiertes Ergebnis: Um die Umverteilung zugunsten der Stromkunden zu organisieren, wird eine einseitige Belastung der Beschäftigten in Kauf genommen. Dabei spielt die Situation der Beschäftigten in der öffentlichen Debatte noch nicht einmal eine Rolle. Es sind vielmehr die monopolähnlichen Profite, welche die Öffentlichkeit auf die Barrikaden treiben. Gleichwohl - oder: umso mehr - sind Politik und Gewerkschaften gefordert, um zu einer ausgewogenen Lastenverteilung zu finden. Die Gewinnbegrenzungskomponente des $\$ 29$ GWB mag zumindest in der Erzeugung sowie im Handel und Vertrieb ein Schritt in die richtige Richtung sein, um dem Profit wieder die Rolle einer Residualgröße zu geben. Zwingende Voraussetzungen für diesen überfälligen Schritt wären eine entsprechend detaillierte, scharf abgegrenzte Definition des angesetzten Kosten- und Gewinnbegriffs und vor allem ausreichende Prüfkapazitäten bei den Kartellbehörden.

Allerdings sind Zweifel angebracht, dass das Primat der Preissenkungen beim Strom gesamtwirtschaftlich und gesellschaftlich sinnvoll ist; besteht doch die Gefahr, dass sinkende Strompreise sowohl die ökologisch zwingend gebotene Energieeinsparung als auch das Etablieren regenerativer Energien erschweren. "So wird die Entlastung der Haushaltskasse mit einer stärkeren Umweltbelastung und einer größeren Abhängigkeit von zukünftigen Preissteigerungen erkauft" (Hennicke/ Müller 2005, S. 145)

10 In dem Umfang, in dem das "eigene" Unternehmen über das „eigene“ Netz infolge des höheren Wettbewerbs weniger Strom liefert, würde die Konkurrenz bei preisunelastischer Gesamtnachfrage mehr durchleiten. 
Angenendt, N./Müller, G./Stronzik, M./Wissner, M. (2007): Stromerzeugung und Stromvertrieb - eine wettbewerbsökonomische Analyse, wikDiskussionsbeiträge Nr. 297

ARegV (2007): Verordnung über die Anreizregulierung der Energieversorgungsnetze vom 29. Oktober (BGBI. I S. 2529)

Baur, J. F./Pritzsche, K. U./Garbers, M. (2006): Anreizregulierung nach dem Energiewirtschaftsgesetz, Baden-Baden

Bontrup, H.-J./Troost, A. (1988): Preisbildung in der Elektrizitätswirtschaft. Ein Beitrag zur Diskussion um die Novellierung der Stromtarife, Bremen

Bundesministerium für Umwelt (2007): Erfahrungsbericht 2007 zum Erneuerbaren-Energie-Gesetz gemäß § 20 EEG - BMU-Entwurf-Kurzfassung vom 05.07.

Bundesnetzagentur (BNetzA) (2008): Bericht gemäß § 63 Abs. 4 EnWG zur Auswertung der Netzzustands- und Netzausbauberichte der deutschen Elektrizitätsübertragungsnetzbetreiber, Bonn

Bundesverband der Energie- und Wasserwirtschaft (bdew) (2008):

Stromwirtschaft modernisiert Kraftwerkspark, Pressemitteilung Januar Bundeswirtschaftsministerium (2008): Wettbewerb im Energiebereich, in: Monatsbericht Januar, S. 24-29

Deutsche Bundesbank (2007): Ertragslage und Finanzierungsverhältnisse deutscher Unternehmen im Jahr 2006, in: Monatsbericht Dezember,

S. 31-55

Deutscher Bundestag (2007): Ausschuss für Wirtschaft und Technologie: GWB-Novelle gegen das Votum von FDP und Grünen angenommen, hibMeldung vom 14.11.

Diekmann, J./Leprich, U./Ziesing, H. J. (2007): Regulierung der Stromnetze in Deutschland. Ökonomische Anreize für Effizienz und Qualität einer zukunftsfähigen Netzinfrastruktur, Düsseldorf

Handelsblatt (1998): Rexrodt: Keine Schutzzäune für Kommunen, Ausg. 4.2., S. 12

Handelsblatt (1998): RWE und Veba sind die Favoriten in der Stromwirtschaft, Ausg. 11.2., S. 29

Handelsblatt (1998): Alleingang beim Stromwettbewerb, Ausg. 2.3., S. 5 Handelsblatt (1998): Strom- und Gaskunden im Wettbewerb umworben, Ausg. 28.4., S. 5

Handelsblatt (2007): Netzagentur kritisiert Strompreiserhöhungen,

Ausg. 8.11., S. 19

Handelsblatt (2007): Barroso stellt sich auf einen erbitterten Kampf ein, Ausg. 20.9., S. 3
Handelsblatt (2008): Fahndung nach dem Zirkusdirektor, Ausg. 6.1., S. 5 Hennicke, P./Müller M. (2005): Weltmacht Energie. Herausforderung für Demokratie und Wohlstand, Stuttgart

Leprich, U. (2007): Die vier großen deutschen Energieunternehmen unter der Lupe (Kurzstudie), Saarbrücken

Monopolkommission (2004): Fünfzehntes Hauptgutachten 2002/2003, Bundestagsdrucksache 15/3610

Monopolkommission (2007a): Monopolkommission, Strom und Gas 2007: Wettbewerbsdefizite und zögerliche Regulierung, Sondergutachten gemäß § 62 Abs. 1 EnWG, o. O.

Monopolkommission (2007b): Preiskontrollen in Energiewirtschaft und Handel? Zur Novellierung des GWB, Sondergutachten gemäß § 44 Abs. 1 Satz 3 und 4 GWB, o. O.

Ockenfels, A. (2007): Strombörse und Marktmacht, in: Energiewirtschaftliche Tagesfragen 5, S. 44-58

Richtlinie Elektrizität 96/92/EG (1996): Richtlinie 96/92/EG des Europäischen Parlaments und des Rates vom 19. Dezember betreffend gemeinsame Vorschriften für den Elektrizitätsbinnenmarkt, 1997 Nr. L 27/20 Schmidt, I. (2005): Wettbewerbspolitik und Kartellrecht, 8. Aufl., Stuttgart Statistisches Bundesamt: Produzierendes Gewerbe, Fachserie 4, Reihe 6.1: Beschäftigung, Umsatz, Investitionen und Kostenstruktur, verschiedene Jahrgänge

Wirtschaftswoche (1998): Strommarkt: Festung Deutschland, Ausg. 12.3., S. 62

Verband kommunaler Unternehmen (VKU) (2006): VKU-Stellungnahme zum Entwurf des Berichtes der Bundesnetzagentur nach § 112a EnWG zur Einführung der Anreizregulierung nach § 21a EnWG vom 02. Mai, Berlin

Vereinte Dienstleistungsgewerkschaft (ver.di) (2008): Für eine Netzentgeltregulierung "mit Augenmaß“, Flugblatt, verteilt auf der Betriebs- und Personalräte-Veranstaltung am 24.01.

Vollhals, J./Schwarz, H.-G./Dees, P./Meier, S. (2007): Struktur und Konzentration im Deutschen Stromendkundenmarkt, in: Energiewirtschaftliche Tagesfragen 1/2, S. 14-16

von Hirschhausen, C./Weigt, H./Zachmann, G. (2007): Preisbildung und Marktmacht auf den Elektrizitätsmärkten in Deutschland. Grundlegende Mechanismen und empirische Evidenz, Studie im Auftrag des Verbandes der Industriellen Energie- und Kraftwirtschaft e. V. (VIK), Electricity Markets Working Papers WP-EM 15 